\title{
Stationary and axisymmetric configurations of compact stars with extremely strong and highly localized magnetic fields
}

\author{
Kotaro Fujisawa*, Shin'ichiro Yoshida and Yoshiharu Eriguchi \\ Department of Earth Science and Astronomy, \\ Graduate School of Arts and Sciences, University of Tokyo, \\ Komaba, Meguro-ku, Tokyo 153-8902, Japan \\ *email: fujisawa@ea.c.u-tokyo.ac.jp
}

\begin{abstract}
Using a new formulation to compute structures of stationary and axisymmetric magnetized barotropic stars in Newtonian gravity, we have succeeded in obtaining numerically exact models of stars with extremely high interior magnetic fields. In this formulation, there appear four arbitrary functions of the magnetic flux function from the integrability conditions among the basic equations. Since in our new formulation these arbitrary functions appear in the expression of the current density, configurations with different current distributions can be specified by choosing the forms of the arbitrary functions.

By choosing appropriate forms for the four arbitrary functions, we have solved many kinds of equilibrium configurations both with poloidal and toroidal magnetic fields. Among them, by choosing special form for the toroidal current density, we have been able to obtain magnetized stars which have extremely strong poloidal magnetic fields deep inside the core region near the symmetric axis. By adopting the appropriate model parameters for the neutron stars, the magnetic fields could be $10^{14} \sim 10^{15} \mathrm{G}$ on the surfaces and be about $10^{17} \mathrm{G}$ in the deep interior regions. For other model parameters appropriate for white dwarfs, the magnetic fields could be around $10^{7} \sim 10^{8} \mathrm{G}$ (surface regions) and $10^{9} \sim 10^{10} \mathrm{G}$ (core regions). It is remarkable that the regions with very strong interior magnetic fields are confined to a very narrow region around the symmetric axis in the central part of the stars. The issues of stability of these configurations and of evolutionary paths to reach such configurations need to be investigated in the future work.
\end{abstract}

Keywords. Stars: interior - Stars: magnetic fields - Stars: neutron - Stars: white dwarfs

\section{Introduction}

According to the recent observations, there are many strong magnetized white dwarfs and neutron stars. Using observations of Zeeman effect and cyclotron absorption or assuming magnetic dipole spin-down, the strength of their magnetic fields at the stellar surfaces has been estimated. For magnetized white dwarfs, the observed magnetic fields on the surfaces range $\sim 3 \times 10^{4}-10^{9} \mathrm{G}$. On the other hand, for neutron stars, they are estimated to be $10^{9}-10^{15} \mathrm{G}$. However, we cannot observe the inner magnetic fields directly and almost nothing has been known about them. Thus, it is a very interesting and important task to know the inner magnetic fields by some other means. One of the possibilities is to construct and study theoretical models in the possible domain of parameter space. To this end, we have extended the Tomimura-Eriguchi formulation (Tomimura \& Eriguchi 2005) to a more general formulation and have developed a new numerical scheme. We have calculated the magnetized equilibria consistently using this new scheme. As a result, we have succeeded in obtaining configurations with extremely strong and highly localized poloidal magnetic fields deep inside the core region near the symmetric axis. 


\section{Formulation}

In this section, we will explain our new formulation briefly. Our formulation is based on Tomimura-Eriguchi scheme (Tomimura \& Eriguchi 2005, Yoshida \& Eriguchi 2006, Yoshida et al. 2006, and Otani et al. 2009).

Assumptions for the magnetized stars are as follows. (1) They are in stationary states, i.e. $\partial / \partial t=0$. (2) They are axisymmetric about the magnetic or the rotational axis, i.e. $\partial / \partial \varphi=0$. (3) When stars are rotating and have magnetic fields, the rotational axis and the magnetic field axis coincide. (4) The stars are self-gravitating. (5) Gravity is Newtonian. (6) The ideal MHD approximation applies. (7) No currents appear in the vacuum region. (8) Barotropic equation of state $(p=p(\rho))$. For neutron stars, we employ the polytropic equation $\left(p=K \rho^{1+1 / N}\right)$ with $N=1$ (Fujisawa et al. 2011a). For white dwarfs, we use the equation of state for completely degenerate gas with zero temperature (Fermi gas) (Fujisawa et al. 2011b).

Under these assumptions, we reduce Maxwell equations, Poisson equation for the gravitational potential $\phi_{g}$ and the equation of motion in the stationary state which reads as follows:

$$
\frac{1}{\rho} \nabla p=-\nabla \phi_{g}-\frac{1}{2} \nabla|\boldsymbol{v}|^{2}+\boldsymbol{v} \times \boldsymbol{\omega}+\frac{1}{\rho}\left(\frac{\boldsymbol{j}}{c} \times \boldsymbol{H}\right),
$$

where variables have their usual meanings. In order to obtain axisymmetric and stationary states of magnetized stars, we introduce the magnetic flux function $\Psi$ as follows:

$$
H_{R} \equiv-\frac{1}{R} \frac{\partial \Psi}{\partial z}, \quad H_{z} \equiv \frac{1}{R} \frac{\partial \Psi}{\partial R} .
$$

Here, we use the cylindrical coordinates $(R, \varphi, z)$. Usually, using this flux function $\Psi$, the Grad-Shafranov (GS) equation has been solved (see e.g. Eq.(19) in Lovelace et al. 1986). However, in our new formulation, instead of solving the GS equation, we derive the elliptical type partial differential equation for the $\varphi$-component of the vector potential $A_{\varphi}(=\Psi / R)$ from the Maxwell equation, i.e. the Ampére's law as follows:

$$
\Delta\left(A_{\varphi} \sin \varphi\right)=-4 \pi \frac{j_{\varphi}}{c} \sin \varphi
$$

Thus, we have two Poisson-type equations. Using Green's functions for the Laplacian, we can take account of the appropriate boundary conditions at infinity and obtain the following integral equations:

$$
\phi_{g}=-G \int \frac{\rho\left(\boldsymbol{r}^{\prime}\right)}{\left|\boldsymbol{r}-\boldsymbol{r}^{\prime}\right|} d^{3} \boldsymbol{r}^{\prime}, \quad A_{\varphi} \sin \varphi=\frac{1}{c} \int \frac{j_{\varphi}\left(\boldsymbol{r}^{\prime}\right)}{\left|\boldsymbol{r}-\boldsymbol{r}^{\prime}\right|} d^{3} \boldsymbol{r}^{\prime}
$$

In order to integrate these equations, we need the distributions of source terms $\rho$ and $j_{\varphi}$. We calculate the density $\rho$ from the first integral of the equation of motion:

$$
\int \frac{d p}{\rho}=-\phi_{g}-\frac{1}{2}|\boldsymbol{v}|^{2}+\int \mu(\Psi) d \Psi+R v_{\varphi} \Omega(\Psi)+C,
$$

and the current density $j_{\varphi}$ from the integrability of the equation of motion:

$$
\frac{\boldsymbol{j}}{c}=\left(\frac{\kappa^{\prime}(\Psi)}{4 \pi}+\frac{Q^{\prime}(\Psi)}{4 \pi} R v_{\varphi}\right) \boldsymbol{H}+\frac{Q(\Psi)}{4 \pi} \boldsymbol{\omega}+\rho R\left(\mu(\Psi)+\Omega^{\prime}(\Psi) R v_{\varphi}\right) \boldsymbol{e}_{\varphi} .
$$

Where, $\kappa, Q, \mu$ and $\Omega$ are arbitrary functions of the flux function $\Psi$. These four arbitrary functions are essentially the same ones as shown in Lovelace et al. (1986). Choosing forms of these arbitrary functions appropriately, we first calculate Eq. (2.5) and Eq. (2.6) and then obtain $\phi_{g}$ and $\Psi$ from Eqs. (2.4). This iteration is cycled until the system 

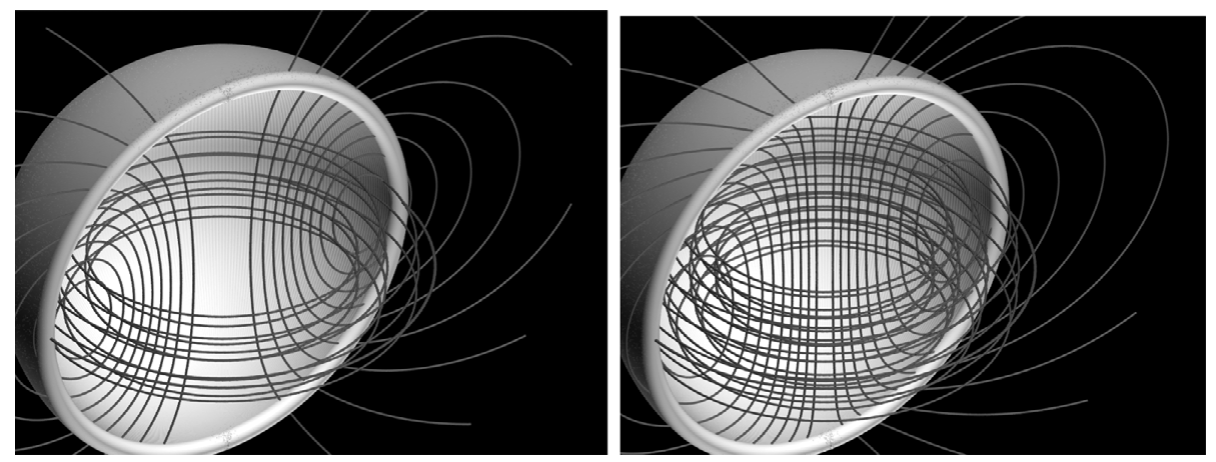

Figure 1. Common features appear in all configurations with both poloidal and toroidal magnetic fields. Configurations with almost dipole distributions of the magnetic fields (left, $m=0.0$ ) and configurations with highly localized and extremely strong magnetic fields (right, $m=-3.0$ ).

settles down to a converged set of physical quantities and we obtain equilibrium states of magnetized stars.

We choose the arbitrary function form as $\mu(\Psi)=\mu_{0}(\Psi+\epsilon)^{m}$ (Fujisawa et al. 2011a). It should be noted that previous investigations such as Tomimura \& Eriguchi (2005) treated only $\mu=\mu_{0}$ (constant) configurations. Employing this functional form, we have obtained various magnetized configurations by changing the values of the parameter $m$.

\section{Results and Discussion}

Fig. 1 shows the magnetic field distributions for the configurations with both poloidal and toroidal magnetic fields. Since almost all of our numerically obtained results have the similar helical structures of the magnetic fields, it is very natural to consider that this kind of magnetic structures are commonly realized in axisymmetric and stationary barotropic equilibrium configurations. Although the equilibrium state in the right panel ( $m=-3.0$ solution) shows a helical field distribution, its poloidal magnetic field is highly localized near the symmetry axis. Thus we will call this highly localized configuration.

In Figure 2, shown are the structure (left panel) and the relative strength of the magnetic multipole moments (right panel) for neutron stars with the parameter $m=0$. In the left panel, the stellar surface, the field lines of the poloidal magnetic fields and the continuous distributions of $\log _{10}|\boldsymbol{H}|$ are shown. In the right panel, the ratios of the strength of the magnetic multipolar moments in the vacuum region to that of the dipole component are displayed. In this figure, the values of the averaged magnetic fields on the stellar surface and in the central region are $H_{\text {sur }} \sim 8.0 \times 10^{14} \mathrm{G}$ and $H_{c} \sim 5.0 \times 10^{15} \mathrm{G}$, respectively. As seen from this figure, higher multipole components are not so large, the magnetic filed of this configuration is described mostly by the dipole moment.

In Figure 3, the same quantities as those in Figure 2 are shown for configurations with the parameter $m=-2.2$. Although we have computed the model so that the value of the surface magnetic field can be almost the same as that for the configuration in Figure 2, i.e. $H_{\text {sur }} \sim 8.0 \times 10^{14} \mathrm{G}$, the poloidal magnetic field of this model is highly localized near the symmetry axis and $H_{c}$ reaches $10^{17} \mathrm{G}$.

It is also remarkable that the contribution of the higher multipole components to the total magnetic fields is appreciable. For instance, the $n=3$ component (oct-pole component) is about $15 \%$ of the dipole component. Moreover, the $n=5$ and $n=$ 7 components are few percents of the dipole component. Therefore, this configuration 


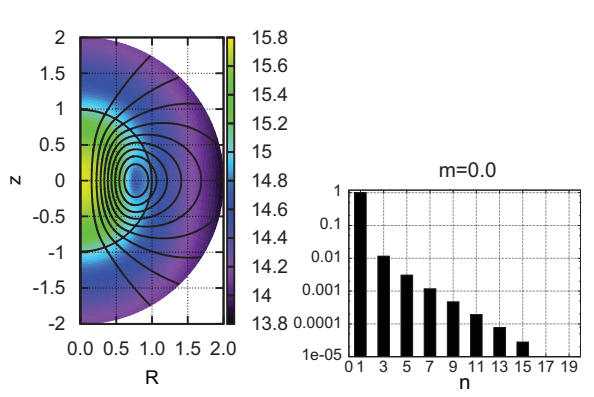

Figure 2. $m=0.0$ configuration. Left: stellar surface line, the poloidal field lines are shown. The distributions of the value of $\log _{10}|\boldsymbol{H}|$ are shown by the continuous map. Right: Ratios of the strength of the magnetic multipolar moments to that of the magnetic dipole moment $(n=1)$ is displayed. The typical values of the magnetic fields are $H_{\text {sur }} \sim 8.0 \times 10^{14} \mathrm{G}$ (surface) and $H_{c} \sim 5.0 \times 10^{15} \mathrm{G}$ (central region).
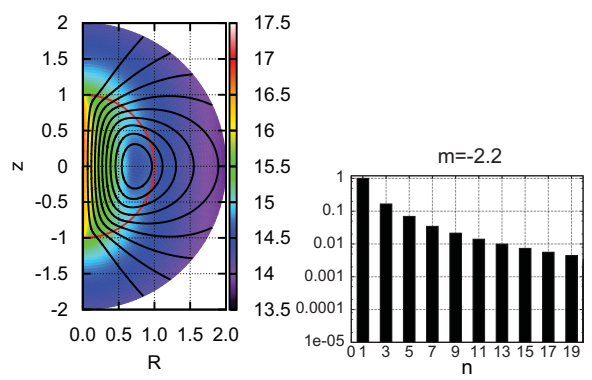

Figure 3. $m=-2.2$ configuration. Left:stellar surface line, the poloidal field lines are shown. The distributions of the value of $\log _{10}|\boldsymbol{H}|$ are shown by the continuous map. Right: Ratios of the strength of the magnetic multipolar moments to that of the magnetic dipole moment $(n=1)$ is displayed. The typical values of the magnetic fields are $H_{\text {sur }} \sim 8.0 \times 10^{14} \mathrm{G}$ (surface) but $H_{c} \sim 1.0 \times 10^{17} \mathrm{G}$ (central region).

cannot be represented by a simple dipole moment configuration but should be considered as a configuration with an extremely strong and highly localized magnetic field.

We have also calculated equilibrium structures of white dwarfs and obtained similar highly localized configurations whose magnetic fields are around $10^{7} \sim 10^{8} \mathrm{G}$ on the surfaces and $10^{9} \sim 10^{10} \mathrm{G}$ in the central core regions (see Fujisawa et al. 2011b).

It would be a very interesting problem to investigate stability natures of our newly obtained magnetized configurations. Magnetic field structures of our configurations are similar to the Twisted-Torus configurations (Braithwaite \& Spruit 2004) which are stable and stationary state reached after a long time evolution. Therefore there is a possibility that our configurations might be stable. However, since we do not have a suitable method to analyze the stability natures of our configurations, we cannot tell exactly whether our configurations are stable or not, at the present time.

\section{Summary}

We have succeeded in obtaining a wide range of axisymmetric and stationary configurations with magnetic fields - from configurations with nearly dipole magnetic fields to those with extremely strong and highly localized magnetic fields. Using the models obtained, we have estimated the strengths of the magnetic fields at the central regions of the stars. We have also shown that the components of magnetic multipolar moments are not always represented by a simple dipole moment model.

\section{References}

Braithwaite, J. \& Spruit, H. C. 2004, Nature, 431, 819

Fujisawa, K., Yoshida, Si., \& Eriguchi, Y., 2011a, in preparation

Fujisawa, K., Yoshida, Si., \& Eriguchi, Y., 2011b, in preparation

Lovelace, R. V. E., Mehanian, C., Mobarry, C. M., \& Sulkanen, M. E., 1986, ApJS, 62, 1

Otani, J., Takahashi, R., \& Eriguchi, Y., 2009, MNRAS, 396, 2152

Tomimura, Y. \& Eriguchi, Y., 2005, MNRAS, 359, 1117

Yoshida, Sj. \& Eriguchi, Y., 2006, ApJ, 164, 156

Yoshida, Sj., Yoshida, Si., \& Eriguchi, Y., 2006, ApJ, 651, 462 